\title{
A Generalized Despeckling Filter for Enhancing Fetal Structures to Aid Automated Obstetric Pathologies
}

\author{
B.Priestly Shan and M.Madheswaran
}

\begin{abstract}
A generalized filter for enhancing fetal structures to aid feature extraction for design of automated clinical diagnosis and decision making system for fetal pathology identification has been presented in this paper. A new class of nonlinear filter, the combinational nonlinear mean median (CNLMM) filter with progressive switching median scheme for speckle detection and maximum likelihood mean estimator for speckle suppression has been proposed. Experimental analysis has been on a database of $\mathbf{1 9 7 8}$ fetal images, obtained from high resolution scanners of various make with various features varying in shape, size and texture. For better analysis, the prominent fetal features have been grouped into linear, angular, curved and homogeneous features. A detailed comparison with various traditional filters has also been made and presented. It has been inferred from the quantitative analysis that the proposed CNLMM filter produces better and stable results compared to its counterparts for all the above feature structures, making it a suitable scheme for speckle suppression.
\end{abstract}

Index Terms - Ultrasound Fetal images, Speckle suppression, Nonlinear filters, CNLMM filters.

\section{INTRODUCTION}

In the recent years, fetal ultrasound images have been identified as one of the useful tool for better diagnosis of prenatal anomalies. Estimation of fetal parameters from diagnostic ultrasound images for fetal anomaly prediction has posed a tough challenge to obstetricians and gynecologists. Fetal growth is to be constantly monitored in subjects with etiology for utero-placental insufficiency, chronic hypertension, renal disease, severe diabetes mellitus and other medical complications such as fetal malnutrition, intrauterine growth retardation or macrosomia [1]. Various sonographic parameters have been measured and reported in the literature for possible prediction of fetal disorders.

Coherent ultrasound imaging systems suffer from speckle noise, creating the acquired images to appear inferior to those 2010

Manuscript received October 19, 2009. Revised and Accepted March

B.Priestly Shan is with the Centre for Advanced Research, Department of Electronics and Communication Engineering, Muthayammal Engineering College, Rasipuram - 637 408, Tamilnadu, India. E-mail priestlyshan@gmail.com.

M.Madheswaran was with PSNA Engineering College and now associated with the Centre for Advanced Research, Department of Electronics and Communication Engineering, Muthayammal Engineering College, Rasipuram - 637 408, Tamilnadu, India. He was also a Research fellow at Banaras Hindu University, Varanasi. E-mail madheswaran.dr@gmail.com. generated by other medical imaging modalities. Speckle noise is a random interference pattern of coherent radiation in a medium containing many sub-resolution scatters. It has been found from the literature that the presence of speckle noise can reduce a physician's ability to detect pathologies by a factor of eight [1-2]. Without speckle, small high-contrast targets, low contrast objects and image texture can be analyzed efficiently. Hence suppression of Speckle from medical ultrasound images represents a critical pre-processing step, providing clinicians with enhanced diagnostic ability. However, it is vital that regions of interests are not compromised during speckle removal. A number of speckle reduction techniques have been developed and reported over the years to reduce speckle in images captured by coherent systems. Speckle suppression techniques cited in the literature can be classified into two categories as multi-look processing (equivalent to lowpass filtering) where several looks obtained from the same scene are averaged and smoothening techniques where the speckle is smoothened after images have been formed. The use of multilook processing to fetal images usually results in poor performance, since it suppresses both the speckle and the texture information of clinical importance at the same time. In the recent past, numerous speckle suppression filters have been proposed for enhancing fetal structures which include the median [2], Lee [3], Kuan [4], Frost [5], Hybrid median [6], SRAD[7], Relaxed median [9], and Homomorphic [10]. Each speckle reduction filter is designed based on different criteria and parameters. The median filter performs spatial filtering in a square-moving window known as the kernel. This filtering is based on the statistical relationship between the central pixel and the surrounding pixels. The Lee filter is based on a linear speckle noise model and a minimum mean square error (MMSE) design approach. The lee filter identifies regions with low and constant variance as areas for noise reduction. In a region with no signal activity, the filter outputs the local mean. In the Kuan Filter [4], the multiplicative noise model is first transformed into a signal-dependent additive noise model. Then the MMSE criterion is then applied to this model. The resulting filter has the same form as the Lee filter but with an enhanced weighting function. The Frost filter differs from the Lee and Kuan filters with respect that the scene reflectivity is estimated by convolving the observed image with the impulse response of the coherent imaging system. The system's impulse response has been calculated by minimizing the mean square error between the observed image and the 
scene reflectivity model which is assumed to be an autoregressive process. These filters have achieved a degree of suppressing speckle while preserving the texture information. These filters represent unique tradeoffs between noise reduction, imaging smoothing, computational complexity, and visual representation. Ultimately, the goal of speckle reduction is to smooth out the image while preserving textural information and structural features, such as edges.

Further, the performances of the filters are also found to vary with different shape features. It has been evident from the literature a filter used for enhancing one feature may not be suitable for speckle suppression of other feature if there is a variation in the shape [2]. For example, the Froast filter, which is effective in enhancing angular edges, has been proved to be inefficient in enhancing curved structures. Few works have been reported by researchers to develop a generalized despeckling scheme for shape features in the recent past. The structures reported for fetal growth estimation can be grouped based on the shape as linear structures, curve shaped structures, spherical structures, homogeneous structures or angular structures. This paper focuses on the development of a generalized optimal filter for suppressing speckle noise from ultrasound fetal images while attempting to retain clinically significant regions of interest. This work proposes a new speckle reduction filter involving a speckle detection step using a progressive switching median filter to mitigate the presence of speckle noise in combination with a maximum likelihood mean estimator for speckle suppression. Simulations suggest that the proposed denoising technique offers superior visual quality, and the quantitative analysis measures [2] [13] such as PSNR, ENL, MAE and IEF are numerically comparable to other speckle suppression filters reported in the literature. Hence this research is expected to improve the quality of clinical ultrasound fetal images, leading to the development of improved automated diagnosis system for clinical decision support systems in the field of obstetrics and gynecology.

\section{Methods AND MATERIALS}

\section{A. Image Acquisition}

The test images are obtained from three different scanning systems, namely Wipro GE Logic 400 Curvilinear probe with transducer frequency of $3-5 \mathrm{MHz}$, THI Siemens machine using curvilinear probe with a transducer frequency of 2.5 $\mathrm{MHz}$ and Toshiba Colour Doppler scan SSD 420A (NEH1030) using a transducer with curvilinear probe containing phased array elements with frequency of 3-5 MHz The fetal images are obtained from subjects at 5-36 weeks of gestation. The images for analysis were obtained from Yuvaraj Scans Salem, Mitra Scans, Salem, CT Scans Marthandam and CSI Mission Hospital, Neyoor. The features to be extracted for gestational age estimation includes the [11 - 12] Yolk Sac Diameter (YSD), Gestational Sac Diameter (GSD) and Crown Rump Length (CRL) in the first trimester, Nasal Bone Length (NBL) and Nuchal Translucency Thickness (NTT) in the mid second trimester and Bi-Parietal Diameter (BPD), Femur Length (FL),
Head/Abdominal circumference (HC/AC) and Trans-Cerebellar Diameter (TCD) in the second and third trimesters. The suitable analysis and implementation of automated clinical decision support system requires images with enhanced features for specific measurement. For example, the measurement of Nasal bone length and Nuchal translucency in the second trimester requires a sonographic image with enhanced Head and Upper Thorax region. For obtaining the images at early trimester, transvaginal scan is being used with intra cavitary probe at a frequency of 9-11 MHz. This type of scanning helps us in obtaining images with high resolution and aids in accurate diagnosis. Similar is the case with other features also. Hence necessary care has been given to preserve the shape, size and gray level distribution as it may obliterate the sonographic content of information. For experimental analysis, a database with 1978 fetal images at various stages of gestation has been created. A sample image from the test image set is shown in Figure 1.

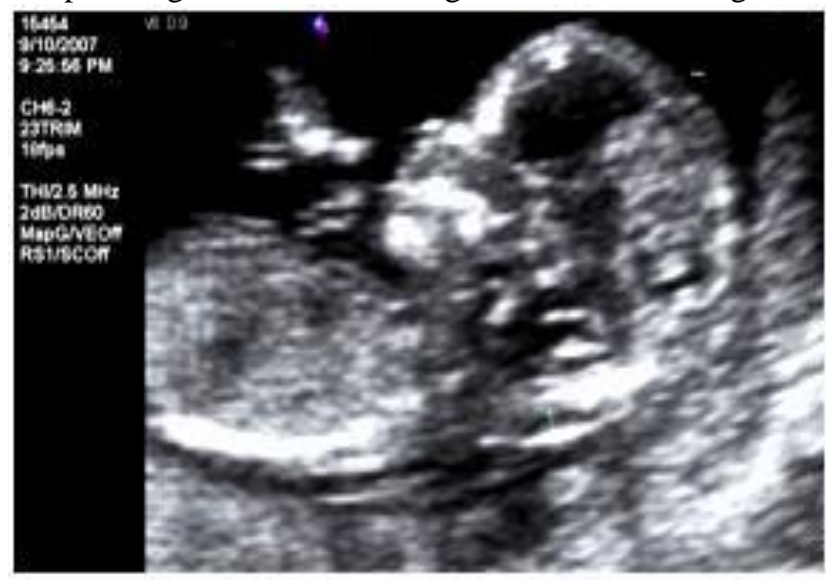

Figure 1: A sample second trimester test image from the developed database of 1978 fetal images showing enhanced upper-thorax and head region

\section{B. Mathematical modeling of CNLMM Filter}

In this paper, speckle suppression has been achieved by a Combinational Non-Linear Mean Median filter (CNLMM).

This filter makes use of an iterative threshold based median filter for speckle detection and a maximum likelihood mean estimator for suppressing the speckle. Traditional despeckling filters using mean or median techniques and their variants, perform spatial filtering in a square-moving window known as the kernel. This filtering is based on the statistical relationship between the central pixel and the surrounding pixels. The mean filter is based on local statistical data given in the filter window. This data is used to determine the noise variance within the central pixel. As the mean filters are implemented uniformly across the image, they tend to modify both noisy as well as good pixels. To avoid damage to the good pixels, a switching scheme has been introduced as reported in [3-7] where noise pixel isolation algorithms are employed before filtering and the detection results are used to decide whether the pixel is to be modified or not. Two image sequences are generated during the speckle detection procedure. The first is a sequence of gray scale images, $\left\{\left\{\mathrm{I}_{\mathrm{i}}^{(0)}\right\},\left\{\mathrm{I}_{\mathrm{i}}^{(1)}\right\},\left\{\mathrm{I}_{\mathrm{i}}^{(2)}\right\},\left\{\mathrm{I}_{\mathrm{i}}^{(3)}\right\} \ldots \ldots\left\{\mathrm{I}_{\mathrm{i}}^{(\mathrm{n})}\right\}\right\}$, where the first sub-image, $\left\{\mathrm{I}_{\mathrm{i}}^{(0)}\right\}$ is the noisy 


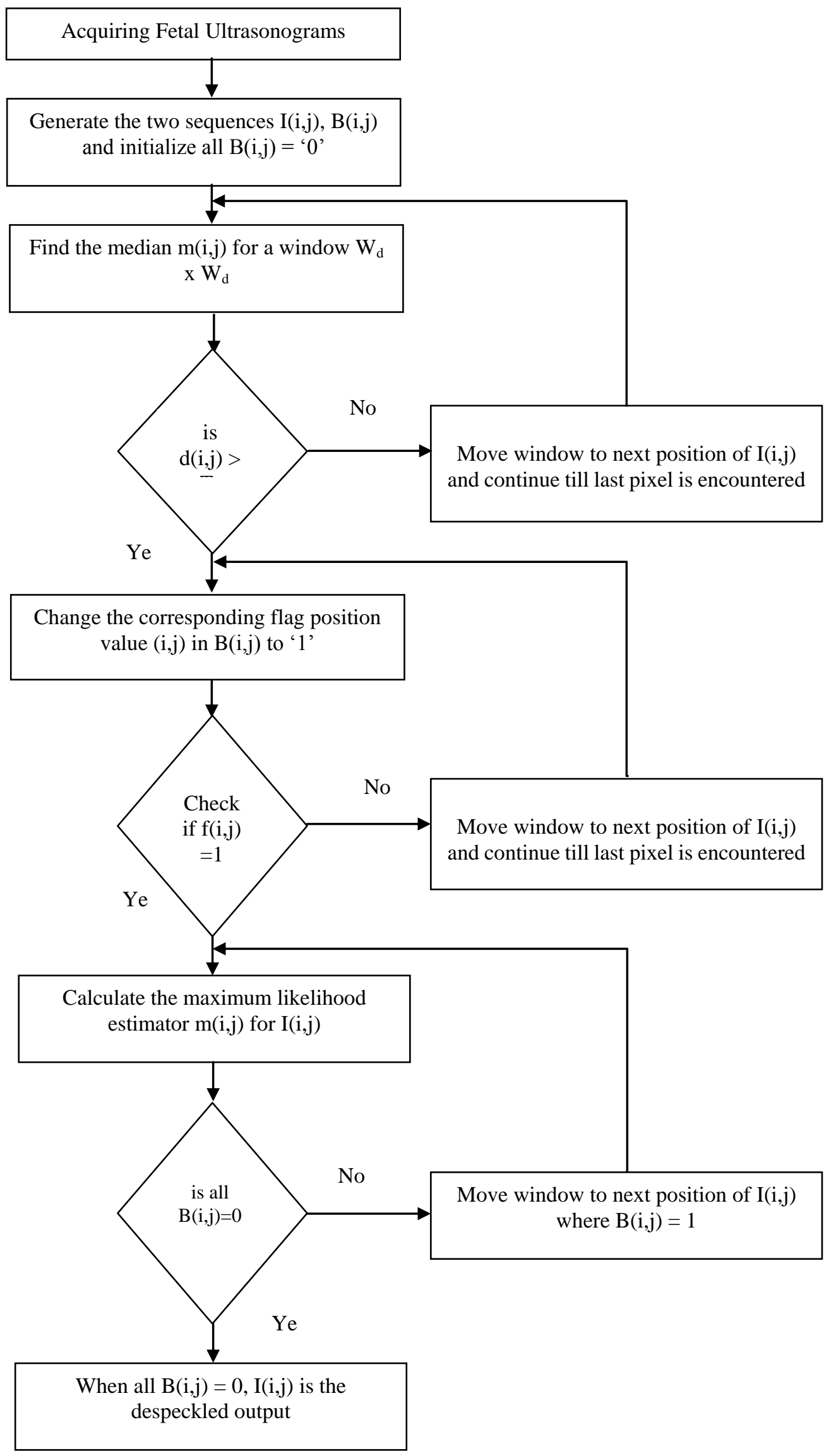

Figure 2: Process flow diagram for speckle detection and suppression 
image to be detected, where ' $\mathrm{I}_{\mathrm{i}}^{(0)}$ ' denotes the pixel value at position $\mathrm{i}=(\mathrm{x}, \mathrm{y})$ in the initial noisy image and ${ }^{\prime} \mathrm{I}_{\mathrm{i}}{ }^{(\mathrm{n})}$ ' represents the pixel value at position $i$ in the image after the nth iteration. The second is a sub-image set, $\left\{\left\{\mathrm{B}_{\mathrm{i}}{ }^{(0)}\right\},\left\{\mathrm{B}_{\mathrm{i}}{ }^{(1)}\right\}\right.$, $\left.\left\{\mathrm{B}_{\mathrm{i}}^{(2)}\right\},\left\{\mathrm{B}_{\mathrm{i}}^{(3)}\right\} \ldots \ldots\left\{\mathrm{B}_{\mathrm{i}}^{(\mathrm{n})}\right\}\right\}$ with de-speckling coefficients, which determines whether the pixel in the first set sub-image is affected by speckle or not. $B_{i}{ }^{(n)}=0$ means the pixel ' $i$ ' is good and $\mathrm{B}_{\mathrm{i}}^{(\mathrm{n})}=1$ means it has been found to be an speckle. Before the first iteration, we assume that all the image pixels are good, i.e., $\mathrm{B}_{\mathrm{i}}{ }^{\mathrm{n})}=0$. For speckle detection, a median filter with a sliding window $S_{w} x S_{w}$ is generated and then the median for the pixel centered about ' $p$ ' is to be determined, where $\mathrm{p}$ is the pixel to be detected as good or bad ( $\mathrm{w}$ is normally an odd number). If $\mathrm{S}_{\mathrm{w}} \mathrm{x} \mathrm{S}_{\mathrm{w}}$ is a window centered about $\mathrm{P}_{(\mathrm{i}, \mathrm{j})}$, then

$$
\mathrm{m}_{\mathrm{i}}^{(\mathrm{n}-1)}=\operatorname{Med}\left\{\mathrm{P}_{\mathrm{j}}^{(\mathrm{n}-1)}, \mathrm{j} € \Psi_{\mathrm{i}}^{\mathrm{Sw}}\right\}
$$

and $\Psi_{\mathrm{i}}^{\mathrm{Sw}}$ is given by $\Psi_{\mathrm{i}}^{\mathrm{Sw}}=\left\{\mathrm{P}_{\mathrm{i}}^{\mathrm{j}+1}, \mathrm{P}_{\mathrm{i}+1}{ }^{\mathrm{j}+1}, \mathrm{P}_{\mathrm{i}+1}{ }^{\mathrm{j}}, \mathrm{P}_{\mathrm{i}+1}{ }^{\mathrm{j}-1}, \mathrm{P}_{\mathrm{i}}^{\mathrm{j}-1}, \mathrm{P}_{\mathrm{i}-1}{ }^{\mathrm{j}}, \mathrm{P}_{\mathrm{i}-1}{ }^{\mathrm{j}+1}, \mathrm{P}_{\mathrm{i}-1}{ }^{\mathrm{j}-1}\right\}$

The speckle detection sub image sequence is generated using the expression given below.

$$
\mathrm{B}_{\mathrm{i}}^{(\mathrm{n})}=\left\{\begin{aligned}
\mathrm{B}_{\mathrm{i}}^{(\mathrm{n})}, & £<T d \\
1, & \text { otherwise }
\end{aligned}\right.
$$

Where ${ }^{£}=m_{i}^{(n-1)}-\Psi_{i}^{S w}$ and $T_{d}$ is the threshold. If the value of $\mathrm{B}_{\mathrm{i}}^{(\mathrm{n})}$ sub-image set or the decision making set is set to 1 or else it is set to 0 . Of the two sets of images, only the second set is utilized for noise filtering process.

Algorithm

Input : $f(x, y)$, the speckled image

Initialize

Window size $\quad$ : $\mathrm{W}_{\mathrm{d}} \mathrm{x} \mathrm{W}_{\mathrm{d}}$ (speckle detection), $\mathrm{W}_{\mathrm{f}} \mathrm{x} \mathrm{W}_{\mathrm{f}}$ (speckle suppression)

No if iterations : 'i' $\rightarrow$ speckle detection, 'j' $\rightarrow$ speckle suppression, ' $\mathrm{k}$ ' $\rightarrow$ entire filter

Flag sequence $: \mathrm{B}_{\mathrm{i}}(\mathrm{n})[:]={ }^{\prime} 0$ ' \% ' 0 ' for good pixel, ' 1 ' for speckle detected pixel

Weighting factor $(\beta) \quad: 0.34$ (determined by trial and error method)

\section{Computations}

\# Speckle Detection \#

Generate flag sequence $B_{i}(n)$ \%initially all pixels are set to be good \%

If median $\mathrm{d}(\mathrm{x}, \mathrm{y})<\mathrm{T}$

else

$$
\mathrm{B}_{\mathrm{i}}(\mathrm{n})[\mathrm{x}, \mathrm{y}]=0
$$

$$
\mathrm{B}_{\mathrm{i}}(\mathrm{n})[\mathrm{x}, \mathrm{y}]=1
$$

Stop when all elements in $\mathrm{f}(\mathrm{x}, \mathrm{y})$ has been classified \#Speckle suppression\#

calculate maximum likelihood estimator at the spatial position $(\mathrm{x}, \mathrm{y})$

$$
\begin{aligned}
& \hat{m}\left(x_{x} y\right)=\hat{m}_{w L}\left(x_{x} y\right)+\beta\left[B\left(x_{x} y\right)-\hat{m}_{w L}\left(x_{x} y\right)\right] \\
& \text { if } \quad B_{i}(n)[x, y]=' 1 ', \quad Y(x, y)=m(x, y) \\
& \text { else if } B_{i}(n)[x, y]=' 0 \text { ', } Y(x, y)=f(x, y)
\end{aligned}
$$

stop when all $\mathrm{B}_{\mathrm{i}}(\mathrm{n})[\mathrm{x}, \mathrm{y}]={ }^{\prime} 0$ '

compute the numerical speckle suppression indicators

PSNR, MAE, ENL, MSE, IEF
The filtering process for speckle suppression is carried out using Maximum likelihood mean estimator filter. The maximum likelihood estimate of the signal can be given by

$$
\widehat{m}_{M L}=-\frac{\sigma^{n}}{2}+\sqrt{\frac{\sigma^{4}}{4}+\frac{1}{N} \sum_{i=1}^{N} X_{i}^{2}}
$$

Where ' $\sigma$ ' is the variance and $X_{i}$ represents the spatial position of the gray level intensity. Based on the above estimate, the signal adaptive maximum likelihood filter for multiplicative Rayleigh noise has been modeled as

$$
\hat{m}(i, j)=\widehat{m}_{M L}(i, j)+\beta(i, j)\left[B(i, j)-\widehat{m}_{M L}(i, j)\right]
$$

Where $B(i, j)$ is the noisy observation at pixel $(i, j)$, $\widehat{m}_{M L}(i, j)$ is the maximum likelihood estimate of $\mathrm{m}(\mathrm{i}, \mathrm{j})$ based on the observations inside the filter window, $\beta(\mathrm{i}, \mathrm{j})$ is a weighting factor and $\widehat{m}\left(i_{j} j\right)$ is the filter output at the pixel $(\mathrm{i}, \mathrm{j})$. The value of the speckle suppressed sequence ${ }_{i}^{(\mathrm{n})}$ is modified only when the pixel ' $i$ ' is a detected as a noisy and $\widehat{m}(i, j)$ is greater than ' 0 '. The sequence $y_{i}^{(n)}$ can be mathematically expressed as

$$
y_{i}^{(n)}= \begin{cases}m_{i}^{(n-1)} & \text { if } B_{i}^{(n-1)}=1 \& \widehat{m}(i, j)>0 \\ y_{i}^{(n-1)} & \text { else }\end{cases}
$$

$y^{(n)}$

$y_{i}$ is again subjected to speckle detection as described above. The process is terminated only when all values in the sequence $B_{i}^{(\mathrm{n})}$ is set to ' 1 '. Thus after the $\mathrm{n}^{\text {th }}$ iteration when all $^{B_{i}^{[n]}}$ is set to ' $1, y_{i}^{[n]}$ is the output speckle suppressed sequence or image.

\section{RESULTS AND DISCUSSION}

Experiments were carried on the fetal image database obtained from ultrasound scanners of different make as discussed earlier. The proposed filter has been utilized for speckle removal for different fetal structures categorized based on shape feature as Angular, Curved, Homogeneous and Linear Structures. For analysis and performance evaluation of the proposed filter, fetal sonograms, obtained from the high resolution scanners were corrupted by speckle noise with variances of $20 \%, 40 \%$ and $50 \%$ which were simulated and added using Matlab. The parameters such as Peak Signal to Ratio (PSNR), Mean Absolute error (MAE), Mean Squared Error (MSE), Image Enhancement Factor (IEF) and Equivalent Number of Looks (ENL) [1] have been used for the quantitative validation of the filter.

The optimal size of the window for the proposed filter has been found to be $3 \times 3$ for normal images. Experiments were also carried out with window sized of $5 \times 5$ and $7 \times 7$. However, for higher levels of corruption, a window size of $5 \times 5$ has resulted in better output. But as the window size is increased, the edges are smoothened, making the automated analysis of fetal structures complex for further processing. Also the 
computational complexity increases as the window size is increased. Hence, for the work reported in this paper, a window size of $3 \times 3$ is used for images with low level of corruption and $5 \times 5$ moving kernel has been used for images corrupted with high level of noise. It has also been inferred that as the value of $\beta$ in the maximum likelihood mean estimator approaches ' 1 ' actual observation is preserved by the suppression of low frequency components and when it
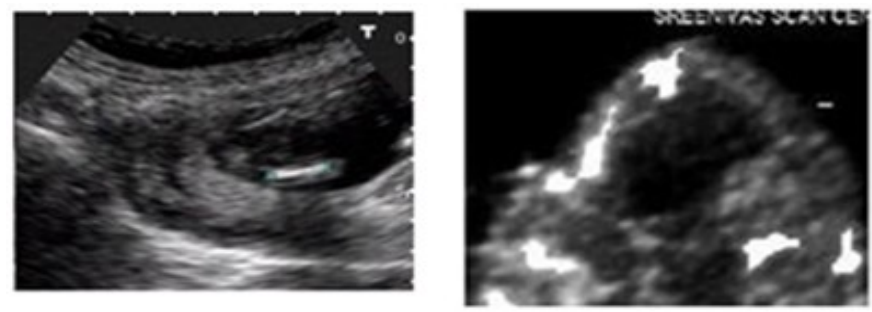

a)linear

(b) Angular

approaches ' 0 ' maximum noise reduction is performed since the high frequency components are suppressed. For experimental purposes, a value of 0.34 for $\beta$ has been identified to be optimal by trial and error method and fixed. From the evaluation of filter parameters, it can be inferred that, not only the edges, but also the diagnostically significant features are also enhanced making it a suitable choice for speckle suppression from ultrasound fetal images.

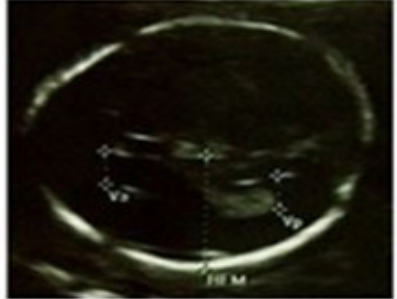

(c) Curved

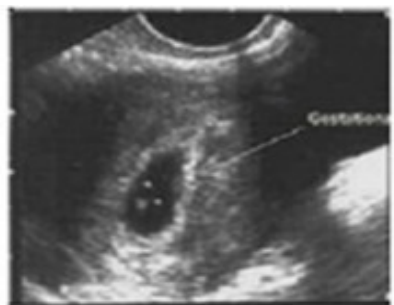

d) Homogeneous

Figure 2 : Test Images from Database of fetal images with various fetal geometric structures (a) Nuchal Translucency with linear homogenous structure (b) Nasal Bone posing angular structure (c) Parietal bones for Bi-Parietal diameter measurement with a ring shaped structure (d) Gestational Sac and Yolk Sac with their homogeneous structures

\section{A. Angular Structure}

The predictors for assessment of the fetal malformation in the second trimester involves extraction of fetal features like nasal bone, Illiac angle and Naso frontal angle. The structures pose angular shape and like the local targets and edges, a sharp angle of an object is also likely to be blurred by speckling artifacts. These features play clinically significant role in prediction of trisomy 21,18 and 13 . Therefore it is important that the shape and edges of the features are preserved while subjecting the image to the despeckling filter The proposed filters ability in preserving the angular structures of fetal sonogram is evaluated experimentally and compared with traditional despeckling filters. Fig 3 shows the experimental results obtained for various filters in despeckling angular structures. The clinical images are harder to decipher due to the large black back ground but the CNLMM filters have shown their ability to despeckle and enhance the clinically significant features as seen from Fig 3. Table1 shows the numerical values of the noise reduction indicators. It can be inferred form the table that both enhanced froast and proposed CNLMM filter perform well in enhancing angular structures. The LEE filter and the relaxed median filters performs the worst in terms of noise suppression. SRAD and Kaun filters are efficient in reducing the speckling artifacts but at the same time tend to smoothen the region of interest making the images appear inferior. The proposed CNLMM filters ability to smooth noise and retain edges as evident from Table 1 and Fig 3, contribute to its superior performance over traditional filters and making it a suitable choice for speckle suppression from clinical ultrasound for angular structures.

\section{B. Curved Structure}

Most fetal structures for gestational age estimation pose round or spherical structures which can generally be grouped as curved structures. These include the Yolk Sac and gestational Sac Measurements in the first trimester, Parietal bones, head circumference and abdominal circumference measurements in the second trimester and third Trimesters. Presence of speckles hinders the edges leading to errors in feature extraction and may introduce errors. Without speckle it may be possible to efficiently predict the gestational age as the underlying objects are enhanced. Experimental results for curved structures were carried out on second trimester images used for BPD measurement. The speckle corrupted ultrasonograms has been denoised using the proposed CNLMM filter and other traditional algorithms. Visual and qualitative assessment suggests that relative to the proposed algorithm, the traditional algorithms except median and relaxed median were not able to suppress the speckle effectively. It can also be inferred from Fig 3 that the output of CNLMM filter is capable of preserving clinical significant areas also while enhancing the parietal bones where as blurring effect can be seen in the resultant images obtained by subjecting the image to SRAD, Kaun and Froast filters. Experiments were conducted with a mask size of $3 \times 3$ and $5 \times 5$ for both speckle detection and speckle suppression schemes. It has been inferred that as the mask size is increased, the blurring effect increases in other filters than CNLMM filters. A mask size of $3 \times 3$ has been found to be optimal. Table 2 shows the numerical values of the noise reduction indicators. Numerical validation shows that the proposed CNLMM filter performs marginally better in terms of PSNR and ENL compared to median, Homomorphic and hybrid median filters for curved structures. It can also be observed that LEE filter and frost filters perform the worst for curved structures. SRAD and Kaun filters effectively despeckle the images but at the same time blur the images making the clinical decision making a complex one. Hence with a mask size of $3 \times 3$ for speckle detection and suppression, the proposed CNLMM filter outperforms the other filters effectively for speckle suppression from curved structures.

\section{Linear Structure}

Due to the multiplicative property of the speckle, the brighter the pixels are, the more will be the speckling artifacts. 
Fetal ultrasonograms for linear feature measurement such as

Nuchal translucency and femur bone are featured with small

high contrast objects lying in a pool of low contrast objects.

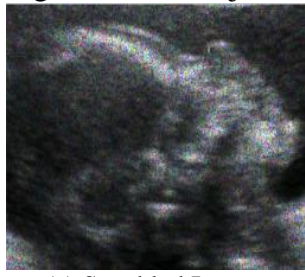

(a) Speckled Image

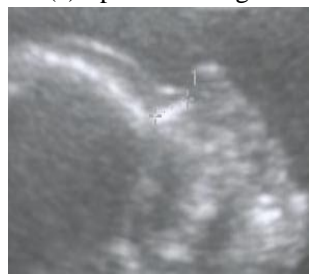

(f) Kaun

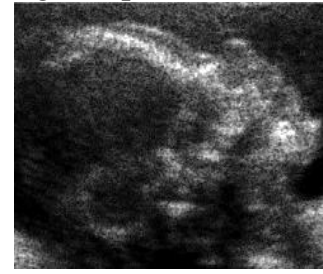

(b) LEE

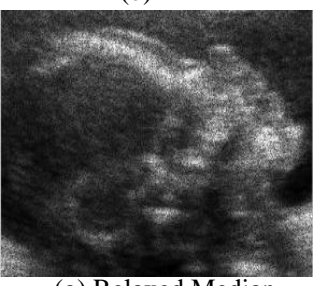

(g) Relaxed Median

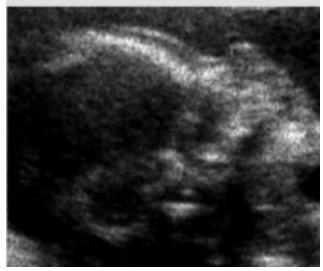

(c) Enhanced Froast

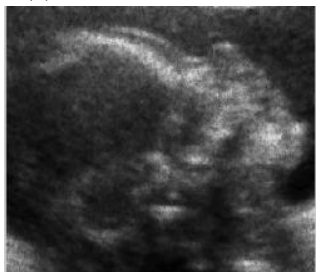

(h) Hybrid Median

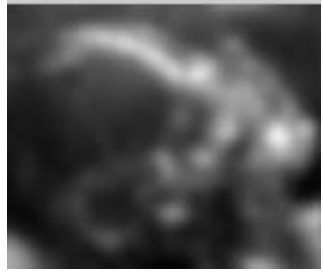

(d) SRAD

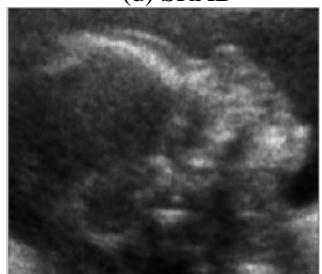

(i) Median

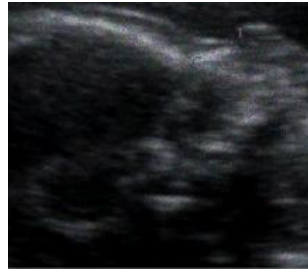

(e) Homomorphic

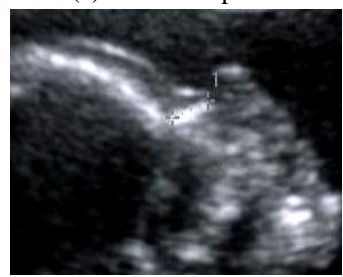

(j) Proposed

Figure 3: Experimental results for denoising angular structures

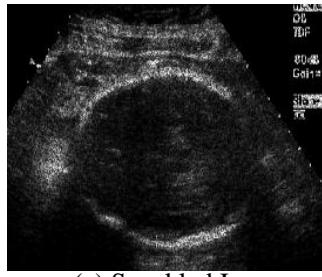

(a) Speckled Image

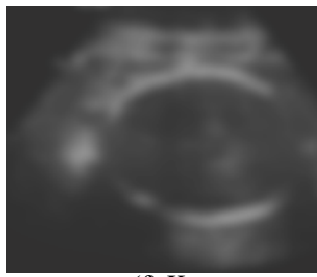

(f) Kaun

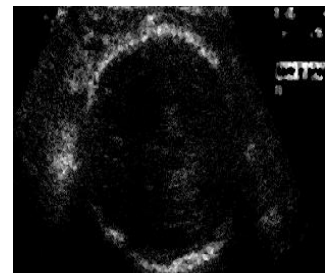

(b) LEE

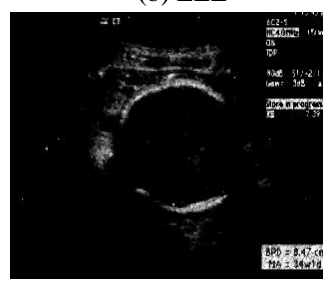

(g) Relaxed Median

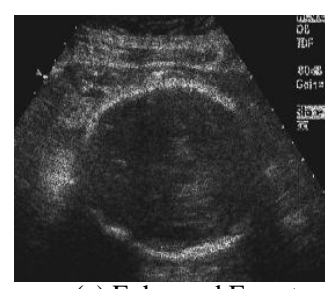

(c) Enhanced Froas

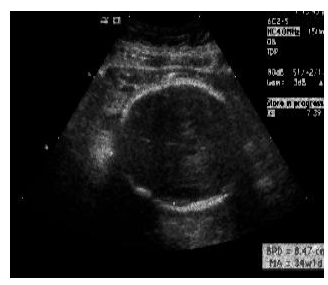

(h) Hybrid Median

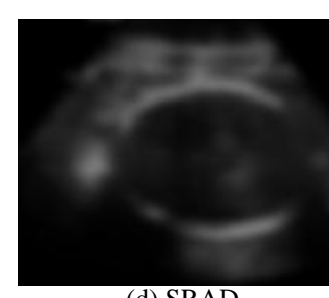

(d) SRAD

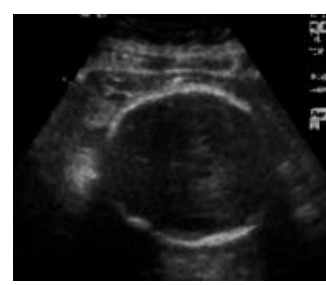

(i) Median

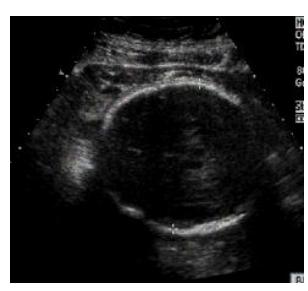

(e) Homomorphic

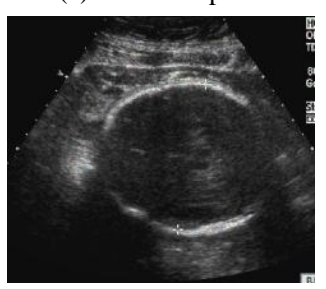

(j) Proposed

Figure 4 : Experimental results for denoising curved structures

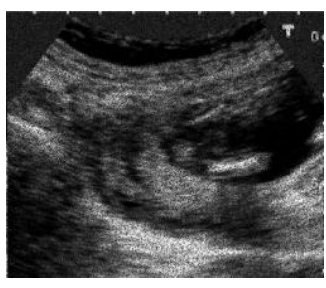

(a) Speckled Image

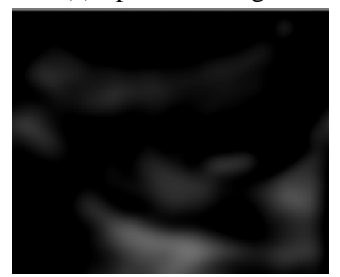

(f) Kaun

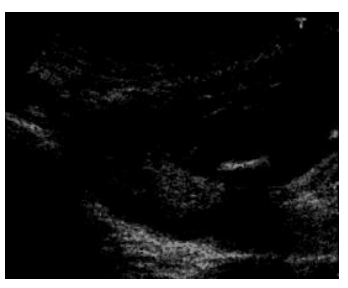

(b) LEE

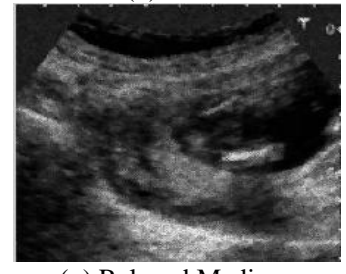

(g) Relaxed Median

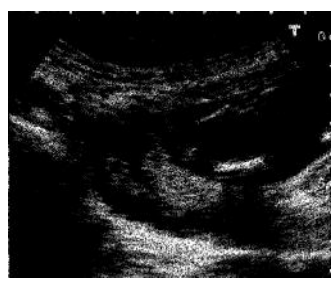

(c) Enhanced Froast

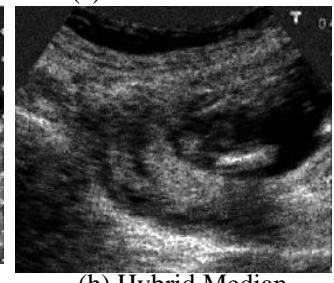

(h) Hybrid Median

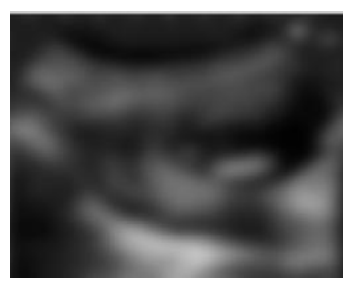

(d) SRAD

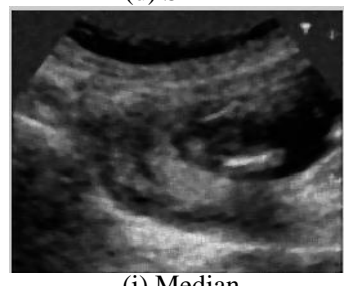

(i) Median

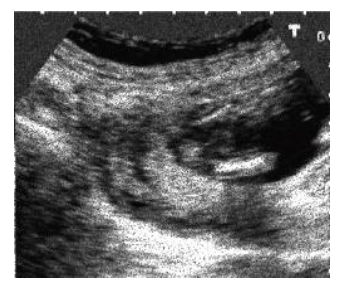

(e) Homomorphic

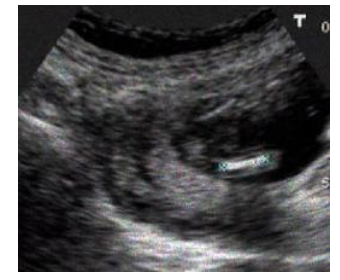

(j) Proposed

Figure 5 : Experimental results for denoising Linear structures

Figures $3-5$ shows the results obtained by processing the speckled images using traditional despeckling filters and the proposed CNLMM filter. The filters used for comparison include the LEE filter, Enhanced Froast Filter, SRAD filter,
Homomorphic filter, Kaun filter, Relaxed Median filter, Hybrid Median filter, median filter and median filter. It can be inferred from the results that proposed CNLMM filter performs slightly better than its counterparts in providing 
stable and reproducible results. Numerical analysis on the

results are tabulated from Tables 1-4. resultant images has been carried out and the quantitative

TABLE 1 : PERFORMANCE EVAlUation MEASURES OF VARIOUS FILTERS FOR ANGULAR StRUCTURES

\begin{tabular}{|c|c|c|c|c|c|}
\hline Filters / Parameters & PSNR & MAE & MSE & IEF & ENL \\
\hline Median [2] & 16.72 & 14.92 & 384 & 6.93 & 2.07 \\
\hline Lee [3] & 14.31 & 12.07 & 432 & 6.41 & 1.721 \\
\hline Kaun [4] & 22.81 & 29.71 & 142 & 8.97 & 2.631 \\
\hline Enhanced Froast [5] & 24.76 & 20.36 & 139 & 9.36 & 2.698 \\
\hline Hybrid Median [6] & 19.32 & 16.31 & 212 & 7.25 & 2.351 \\
\hline SRAD [7] & 16.14 & 13.37 & 357 & 7.02 & 1.876 \\
\hline Relaxed Median [11] & 14.64 & 12.83 & 396 & 6.52 & 1.927 \\
\hline Homomorphic [12] & 18.92 & 16.97 & 304 & 7.76 & 2.342 \\
\hline CNLMM (proposed) & 26.43 & 20.93 & 112 & 9.71 & 2.742 \\
\hline
\end{tabular}

TABLE 2 : PERFORMANCE EVALUATION MEASURES OF VARIOUS FILTERS FOR CURVED STRUCTURES

\begin{tabular}{|l|c|c|c|c|c|}
\hline \multicolumn{1}{|c|}{ Filters / Parameters } & PSNR & MAE & MSE & IEF & ENL \\
\hline Median [2] & 29.07 & 20.96 & 141 & 2.89 & 2.891 \\
\hline Lee [3] & 16.71 & 16.97 & 329 & 7.24 & 1.891 \\
\hline Kaun [4] & 26.92 & 18.92 & 276 & 8.52 & 2.541 \\
\hline Enhanced Froast [5] & 15.62 & 16.42 & 332 & 6.57 & 1.531 \\
\hline Hybrid Median [6] & 28.91 & 17.06 & 413 & 2.07 & 2.072 \\
\hline SRAD [7] & 25.97 & 18.71 & 241 & 7.91 & 1.726 \\
\hline Relaxed Median [11] & 24.31 & 18.61 & 217 & 8.34 & 2.321 \\
\hline Homomorphic [12] & 29.31 & 20.85 & 164 & 9.51 & 2.621 \\
\hline CNLMM (proposed) & 29.462 & 21.71 & 107 & 9.62 & 2.972 \\
\hline
\end{tabular}

TABLE 3 : PERFORMANCE EVALUATION MEASURES OF VARIOUS FILTERS FOR LINEAR STRUCTURES

\begin{tabular}{|l|c|c|c|c|c|}
\hline \multicolumn{1}{|c|}{ Filters / Parameters } & PSNR & MAE & MSE & IEF & ENL \\
\hline Median [2] & 31.07 & 24.36 & 103 & 13.7 & 4.707 \\
\hline Lee [3] & 21.61 & 19.46 & 244 & 9.76 & 3.562 \\
\hline Kaun [4] & 25.62 & 21.62 & 176 & 12.62 & 4.367 \\
\hline Enhanced Froast [5] & 21.07 & 17.31 & 376 & 9.72 & 3.091 \\
\hline Hybrid Median [6] & 20.02 & 19.52 & 274 & 9.52 & 3.921 \\
\hline SRAD [7] & 26.37 & 21.41 & 240 & 11.91 & 4.212 \\
\hline Relaxed Median [11] & 30.96 & 23.91 & 130 & 13.41 & 4.524 \\
\hline Homomorphic [12] & 29.92 & 20.97 & 214 & 11.78 & 4.342 \\
\hline CNLMM (proposed) & 31.32 & 24.76 & 86 & 14.6 & 4.721 \\
\hline
\end{tabular}

TABLE 4: PerformanCe EVAluation MEASURES OF VARIOUS FILTERS FOR HOMOGENEOUS STRUCTURES

\begin{tabular}{|l|c|c|c|c|c|}
\hline Filters / Parameters & PSNR & MAE & MSE & IEF & ENL \\
\hline Median [2] & 24.69 & 21.36 & 251 & 8.27 & 1.927 \\
\hline Lee [3] & 19.62 & 19.72 & 342 & 6.52 & 1.651 \\
\hline Kaun [4] & 24.32 & 21.06 & 176 & 8.21 & 1.976 \\
\hline Enhanced Froast [5] & 21.46 & 19.61 & 321 & 7.06 & 1.706 \\
\hline Hybrid Median [6] & 24.12 & 20.71 & 274 & 7.37 & 1.762 \\
\hline SRAD [7] & 23.72 & 21.52 & 393 & 8.21 & 2.334 \\
\hline $\begin{array}{l}\text { Relaxed Median } \\
\text { [11] }\end{array}$ & 23.96 & 20.97 & 249 & 7.12 & 1.797 \\
\hline Homomorphic [12] & 24.07 & 20.91 & 241 & 7.84 & 1.621 \\
\hline $\begin{array}{l}\text { CNLMM } \\
\text { (proposed) }\end{array}$ & 24.71 & 21.62 & 146 & 8.26 & 2.476 \\
\hline
\end{tabular}

PSNR : Peak Signal to Noise Ratio

MAE : Mean Absolute Error

MSE : Mean Squared Error

IEF : Image Enhancement Factor

ENL : Equivalent Number of Looks 


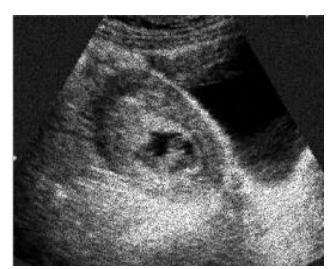

(a) Speckled Image

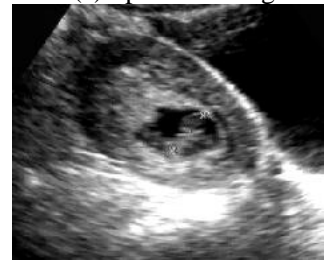

(f) Kaun

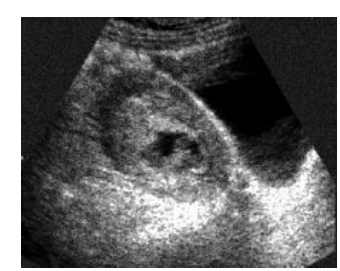

(b) LEE

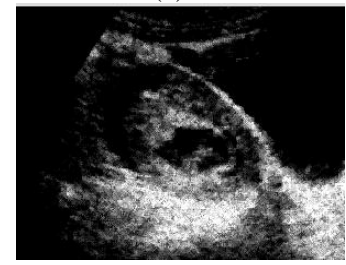

(g) Relaxed Median

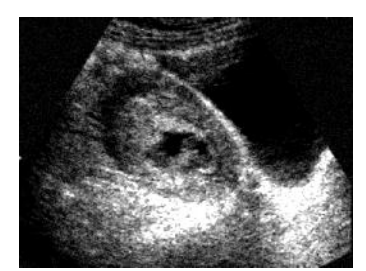

(c) Enhanced Froast

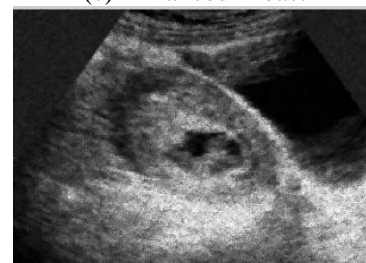

(h) Hybrid Median

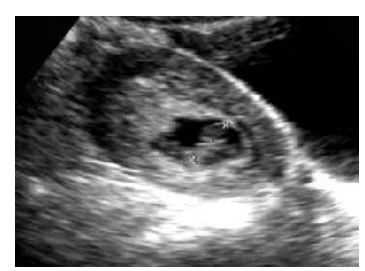

(d) SRAD

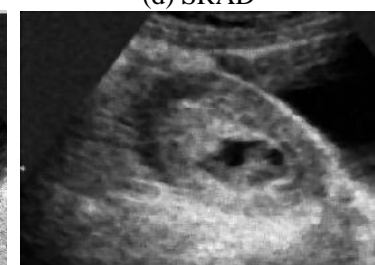

(i) Median

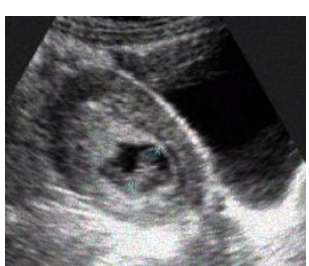

(e) Homomorphic

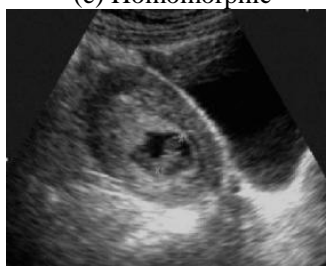

(j) Proposed
Fig 5 shows speckle noise reduction on an ultrasound image with linear structures. It can be inferred that the proposed filter de-noises and preserves the shape much effectively compared to its counterparts. The performance of median, enhanced froast and homomorphic filters has also found to be quite similar in enhancing linear structures.

Table 3 shows the numerical values of the noise reduction indicators. Overall, the CNLMM filter algong with the median and relaxed median filters produced the best PSNR with ENL showing almost similar values. Same was the case with other parameters also. As with the other cases, SRAD filter was effective in suppressing the speckle but resulted in blurring making the output images look inferior. Homomorphic filters also produced the best results for linear structures comparable with CNLMM filters. But with a PSNR of 31.31 and ENL of 4.721, the CNLMM filters clearly outperformed the traditional filters making it a better choice for linear structure speckle suppression schemes.

\section{Homogeneous Structures}

Features for estimating the fetal growth retardation such as Crump Rump Length, Gestational Sac, OFD require segmentation of homogeneous structures. As speckle noise are uniformly distributed throughout the image, analysis of these images become difficult. Fig 6 shows the outputs of de-noised images of various standard filters in comparison to the CNLMM filter for a generally homogenous feature, the crown rump length. The resultant images are difficult to interpret as the eye may not be able to differentiate small high contrast objects surrounded by a number of low contrast features and the improvements are not as clearly evident. Hence it is necessary to fall back on the numerical analysis to provide an indication for the speckle noise reduction effectiveness. The images show that the homomorphic filter and the CNLMM filters are effective in suppressing the speckle and edges more prominently than other filters. The median filter shows very poor results due to over-smoothing while the lee filter tends to under-smooth images.

Table 4 shows the numerical values of the noise reduction indicators. From the table it can be inferred that the , the Kuan filter showed the best results in terms of numerical values with the median filter. However, the images show that the kuan filter does not have good resolution near the edges. This result is due to the large homogeneous regions, causing filters that tend to smooth images to perform slightly better than others.

Based on the visual inspection of the above images and numerical statistics, it can be concluded that

1) The proposed CNLMM filters shows the best performance overall in terms of PSNR, MAE, MSE, IEF values, ENL and visual performance, particularly in cases of edge preservation, high contrast images enhancement and smoothing.

2) The performance of the homomorphic filters for linear, curved and homogeneous structures are comparable with CNLMM filters but gives poor results when trying to despeckle angular structures.

3) Analysis show that the Enhanced Frost, Relaxed Median, Hybrid Median, Median are quite similar

4) The SRAD filter and Kaun filter has despeckled the artifacts but tends to smoothed the edges making the resultant images to look inferior.

5) The Lee filters performed the poorest based on visual comparison and numerical analysis.

In general, all filters have a very good ability to smooth out the noise. However, it can seen that only certain filters retain edges correctly. The quantitative performance measures for the Median, Relaxed median, Hybrid Median, Kuan, Frost, Lee, Homomorphic, SRAD and CNLMM filters have been analyzed. Overall, the best PSNR is provided by the CNMM filter with a mask of $3 \times 3$ for speckle detection and $3 \times 3$ for speckle suppression. The value of $\beta$ has been set to 0.34 . In all cases, the CNLMM filter outperforms the standard ones especially for the low spectral content image with high level noise. The results suggest that the new technique has a large potential in assisting segmentation techniques and automated area/volume calculation methods due to its superior filter performance.

\section{CONClusion}

A new class of nonlinear speckle suppression has been developed and presented in this paper. It can be inferred that the proposed filtering technique preserves the edge information as well as enhance areas of clinical importance with diagnostic information. Analysis has shown that this filter has the ability to decipher speckle noise effectively compared to its counterparts. The ability to despeckle 
speckling artifacts and preserve the edges with stable results for all shape features discussed, makes CNLMM filters a better choice for preprocessing the clinical fetal ultrasonograms. Using CNLMM filter, as the shape features has been preserved, the prediction accuracy of clinical decision support systems for fetal pathology identification can be improved and hence can effectively act as a secondary observer for obstetricians and gynecologist.

\section{REFERENCES}

[1] G Edund Hui-on Ng, "Speckle Noise Reduction Via Homomorphic Elliptical Threshold Rotations in Complex Wavelet Domain", A MS Thesis Presented to University of Waterloo, Canada, 2005.

[2] O Z.Shi and K.B.Fung, "A Comparision of Digital Speckle Filters" in proc. IEEE on IGARSS, Vol 4, pp. 2129 - 2133, August 1994.

[3] J.S Lee, "Refined Filtering of Image Noise using Local Statistics", Journal of Computer Graphic and Image Processing, Vol 15, pp. $380-$ 389,1980 .

[4] D.Kaun, A.Swachuk, T.Strand and P.Chavel, "Adaptive noise smoothening filter for images with signal-independent noise", Journal of Computer Graphic and Image Processing, Vol 7, no 2, March 1985

[5] V.Froast, J.Stiles, K.Shanmugam, and J.Holtzman, "A model for radar images and its application to adaptive digital filtering of multiplicative noise", Journal of Computer Graphic and Image Processing, Vol 4, no 2 , March 1982.

[6] S. Mansoor Roomi, I.M.Lekshmi, V Abhai Kumar, "A Recursive Gaussian Weighted Filter for Impulse Noise Removal” GVIP Journal, Volume 6, Issue 3, pp: 33 - 37 December 2006.

[7] S Khaled, Abou-Bakr, Yaser, "Real Time Speckle Reduction and Coherence enhancement in ultrasound imaging via nonlinear anisotropic diffusion", IEEE Transactions on Bio Medical Engineering, Vol 49, No 9, September 2002.

[8] Alin Achim, Anastasios, Panaigiotis, "Novel Bayesian Multiscale method for Speckle removal in ultrasound images" IEEE Transactions on Medical Imaging, Vol 20, No 8, August 2001.

[9] Zohu Wang and David Zhang, "Progressive switching Median filter for the removal of impulse noise from highly corrupted images", IEEE Transaction on circuits and Systems - II, Analog and Digital Signal Processing, Vol 46, No 1, January 1999.

[10] Alin Achim, Anastasios Bezerianos and Panagiotis, "An Alpha-Stable based Bayesian algorithm for speckle noise removal in the wavelet domain" Proc of NISP - 2001, Baltimore, Maryland USA, June 01 - 03, 2001.

[11] A.C.Volker, P.Zakharov, B,Webber., et.al., "Laser Speckle Imaging with an active noise reduction scheme" Journal of Optic Express, Vol 13, No 24, November 2005.

[12] Sudha, G.R.Suresh, R.Sukanesh, "Wavelet based image de-noising using adaptive sub-band thresholding", International Journal of Soft Computing, Volume 2, Issue 5, PP: 628-632, 2007.

[13] Callen, "Ultrasonography in obstetrics and gynecology", W.B.Saunders Company, Harcourt Brace Jovanovich, Inc 2nd edition,1998, ISBN 0-7216-2244-5.

[14] Gustavo Carneiro, Ph.D., Bogdan Georgescu, Ph.D., Sara Good, RDMS," Knowledge-based Automated Fetal Biometrics", Whitepaper, ACUSON S2000, Siemens Medical Solutions, September 2008.

[15] V. Dutt and J. F. Greenleaf, "Adaptive speckle reduction filter for logcompressed B-scan images,” IEEE Trans. Med. Imag., vol. 15, no. 6 pp. 802-813, Dec. 1996.

[16] Cea.J, "Problems of shape optimal design," Optimization of Distributed Parameters Structures - Ed. E.J.Haug and J.C'ea, Sijthoff and Noordhoff, Alphen aan den Rijn, no. II, pp. 1005-1048, 1981. areas include Biomedical Engineering, Signal Processing and Image Processing. $\mathrm{He}$ is a member of various professional societies including Institute of Electrical and Electronics Engineering (IEEE), International Association of Computer Science and Information Technology (IASCIT), Singapore, Institute of Electronics and Telecommunication Engineering (IETE) and International Brain Research Organization (IBRO). At present he is the secretary of IEEE India council Circuits and Systems Society Chapter. $\mathrm{He}$ is also the Executive member for IEEE India council Electron Devices Society chapter and Solid State Circuits and Systems Society Chapter. $\mathrm{He}$ has received the Outstanding Student Support award in the year 2006 from IEEE USA. He has organized Three Conferences as Coordinator Sponsored by AICTE (2008), IEEEE (2009, 2010). He has also coordinated various Faculty Development Programmes and Short Term Training Programmes in the field of Signal and Image Processing.

M.Madheswaran received the BE Degree from Madurai Kamaraj University in 1990, ME Degree from Birla Institute of Technology, Mesra, Ranchi, India in 1992, both in Electronics and Communication Engineering. $\mathrm{He}$ obtained his $\mathrm{PhD}$ degree in Electronics Engineering from the Institute of Technology,Banaras Hindu University, Varanasi, India, in 1999. At present he is a Principal of Muthayammal Engineering College, Rasipuram, India He has authored over one hundred and twenty five publications in international and national journals and conferences. Currently he is the chairman of IEEE India Electron Devices Society Chapter and Vice Chair of IEEE India Circuits and System Chapter. His areas of interest are theoretical modeling and simulation of high-speed semiconductor devices for integrated optoelectronics application, Bio-optics and Bio-signal Processing and Clinical Decission Support Systems development. He was awarded the Young Scientist Fellowship (YSF) by the State Council for Science and Technology,TamilNadu, in 1994 and Senior Research Fellowship (SRF) by the Council of Scientific and Industrial Research (CSIR), Government of India in 1996. Also he has received YSF from SERC, Department of Science and Technology, Govt. of India. He is named in Marquis Who's Who in Science and engineering in the year 2006. He is a fellow of IETE and IE (India), life member of ISTE and and also a senior member of IEEE., He is also the member of International Association of Computer Science and Information Technology (IACSIT) Singapore.

B.Priestly Shan received his Bachelors Degree in Electronics and Communication Engineering from Manonmaniam Sundaranar University, Tirunelveli, India in the year 2003 and Masters Degree in Communication Systems from Anna university Chennai, India in the year 2006. At present, he is affiliated with the Center for Advanced Research, Department of Electronics and Communication Engineering of Muthayammal Engineering College, Rasipuram. He has authored over 20 research papers in various International/National conferences, Journals and symposiums. His research 